\title{
When upper conditional probabilities are conditional possibility measures
}

\author{
G. Coletti ${ }^{1}$ D. Petturiti ${ }^{1}$ B. Vantaggi ${ }^{2}$ \\ ${ }^{1}$ Dip. Matematica e Informatica, Università di Perugia, Italy \\ ${ }^{2}$ Dip. S.B.A.I., Università di Roma "La Sapienza", Italy
}

\begin{abstract}
Conditioning for (non-additive) uncertainty measures is still an open problem. These measures can arise through probabilistic inference procedures, as in the case of possibility measures, that can be seen as the upper envelope of the extensions of a probability when the corresponding algebras are weakly logically independent. The aim of this paper is to define a conditioning rule (B-conditioning) such that the upper envelope of the extensions of a full conditional probability on an algebra $\mathcal{A}$ is a full Bconditional possibility on another algebra $\mathcal{A}^{\prime}$ under weak logical independence of $\mathcal{A}, \mathcal{A}^{\prime}$.
\end{abstract}

Keywords: Conditional possibility, conditional probability, inference, upper envelope, weak logical independence, generalized Bayesian rule

\section{Introduction}

Prior probabilities have a central role in Bayesian statistics, even if sometimes the elicitation of the prior is not an easy task. For this, in some applications (for instance, in the reconstruction of images or signals) instead of assessing a single prior probability, one can select a suitable family of priors. These probabilities can derive also from a probabilistic information concerning events different from those of interest. Indeed, in some situations it could be necessary to extend to a family of (conditional) events a conditional probability defined on a different domain.

Hence, in order to manage sets of probabilities, it is fundamental to work with their lower and upper envelopes and to make inference through them. These lower and upper envelopes are generally not additive and sometimes they reveal to be measures belonging to well-known families (e.g., plausibility functions $[12,20]$ and possibility measures [14]) which have received a lot of attention also in fuzzy set theory. Nevertheless, as discussed at length in probability theory, to make inference when the conditioning event has measure 0 we need to consider as prior a conditional measure (see, for instance, [8] where conditional probabilities are used in order to interpret a membership function), thus the notion of conditioning for non-additive measures is crucial.

On the other hand, the debate on the conditioning for non-additive measures is still an open issue, as one can verify by the related quite extended literature where several conditioning notions are given (see, e.g., $[4,7,12,9,14,16]$ ).

A first aspect in which the given conditioning notions differ is how the conditional measure is defined: essentially, either starting from a nonadditive unconditional measure through a suitable mathematical rule or directly as a function on a structured domain, satisfying a set of axioms.

Another peculiarity is the relationship between probability theory and the chosen family of nonadditive measures. For example, a well-known correspondence between probabilities and plausibility functions has been recognized in the extension of a probability [17]. On the same line, Dubois and Prade in [15] provided a characteristic property for an upper probability to coincide with a possibility measure, which led in [6] to characterize possibility measures as upper envelopes of the extensions of a probability when a logical condition (weak logical independence) holds.

In this paper we focus on possibility measures on a finite algebra and we introduce the notion of Bconditioning. It is shown that under weak logical independence of two finite algebras $\mathcal{A}, \mathcal{A}^{\prime}$, the upper envelope of the conditional probabilities extending a given full conditional probability $P$ on $\mathcal{A} \times \mathcal{A}^{0}$ is a full B-conditional possibility on $\mathcal{A}^{\prime} \times \mathcal{A}^{\prime 0}$. Furthermore, for any full B-conditional possibility measure $\Pi_{B}$ there exists a full conditional probability $P$ such that the upper envelope of its extensions coincides with $\Pi_{B}$.

\section{Conditional probability and its enlargement}

Let $\mathcal{A}$ be a Boolean algebra of events E's and denote with $(\cdot)^{c}, \vee$ and $\wedge$ the usual Boolean operations of contrary, disjunction and conjunction, respectively. The sure event $\Omega$ and the impossible event $\emptyset$ coincide, respectively, with the top and bottom elements of $\mathcal{A}$ endowed with the partial order $\subseteq$ of implication.

A conditional event $E \mid H$ is an ordered pair of events $(E, H)$ with $H \neq \emptyset$, where the conditional event $E \mid \Omega$ is customarily identified with $E$. Let $\mathcal{H}$ be an additive class (i.e., a set of events closed under finite disjunctions) such that $\mathcal{H} \subseteq \mathcal{A}^{0}=\mathcal{A} \backslash\{\emptyset\}$. An arbitrary set of conditional events $\mathcal{G}=\left\{E_{i} \mid H_{i}\right\}_{i \in I}$ 
can always be embedded into a minimal structured set $\mathcal{A} \times \mathcal{H}$, where $\mathcal{A}=\left\langle\left\{E_{i}, H_{i}\right\}_{i \in I}\right\rangle$ is the Boolean algebra generated by $\left\{E_{i}, H_{i}\right\}_{i \in I}$ and $\mathcal{H}$ is obtained closing $\left\{H_{i}\right\}_{i \in I}$ under finite disjunctions. In the following such set $\mathcal{A} \times \mathcal{H}$ is denoted as $\langle\langle\mathcal{G}\rangle\rangle$.

We refer to the axiomatic definition of conditional probability [5], essentially due to de Finetti [11], Rényi [19] and Dubins [13]. According to it, a real function $P(\cdot \mid \cdot)$ on a structured set $\mathcal{A} \times \mathcal{H}$ of conditional events is asked to satisfy

(i) $P(E \mid H)=P(E \wedge H \mid H)$, for every $E \in \mathcal{A}$ and $H \in \mathcal{H}$

(ii) $P(\cdot \mid H)$ is a finitely additive probability on $\mathcal{A}$, for any $H \in \mathcal{H}$;

(iii) $P(E \wedge F \mid H)=P(E \mid H) \cdot P(F \mid E \wedge H)$, for every $H, E \wedge H \in \mathcal{H}$ and $E, F \in \mathcal{A}$.

Following [13], we say that a conditional probability $P(\cdot \mid \cdot)$ is full on $\mathcal{A}$ if $\mathcal{H}=\mathcal{A}^{0}$, i.e., if it is defined on $\mathcal{A} \times \mathcal{A}^{0}$. Moreover, we simply write $P(E)=P(E \mid \Omega)$.

When $\mathcal{A}$ is finite (as it will always be in this paper), a full conditional probability $P(\cdot \mid \cdot)$ on $\mathcal{A}$ is completely singled out by a linearly ordered class $\left\{P_{0}, \ldots, P_{k}\right\}$ of probability measures on $\mathcal{A}$ whose supports form a partition of $\Omega$. For any $E \mid H \in$ $\mathcal{A} \times \mathcal{A}^{0}$ there exists $\alpha \in\{0, \ldots, k\}$ with $P_{\alpha}(H)>0$ and $P_{\beta}(H)=0$ for $\beta<\alpha$ and $P(E \mid H)=\frac{P_{\alpha}(E \wedge H)}{P_{\alpha}(H)}$.

The class $\left\{P_{0}, \ldots, P_{k}\right\}$ is said to agree with $P(\cdot \mid \cdot)$. This representation is unique when $P(\cdot \mid \cdot)$ is full, while for a non-full conditional probability (i.e., when $\mathcal{H} \subset \mathcal{A}^{0}$ ), there can be several agreeing classes of probabilities on $\mathcal{A}$, each representing a full conditional probability $\tilde{P}$ on $\mathcal{A}$ extending $P$.

Indeed, by the conditional version of the fundamental theorem for probabilities [11], any conditional probability $P$ on $\mathcal{A} \times \mathcal{H}$ can be extended as a conditional probability on $\left\langle\left\langle\mathcal{G}^{\prime}\right\rangle\right\rangle$ where $\mathcal{G}^{\prime} \supseteq \mathcal{A} \times \mathcal{H}$ is an arbitrary superset of conditional events. The extension is generally not unique, in particular, if $\mathcal{G}^{\prime}=(\mathcal{A} \times \mathcal{H}) \cup\{E \mid H\}$ the possible values for the conditional probability of $E \mid H$ range in a closed interval $[\underline{P}(E \mid H), \bar{P}(E \mid H)]$.

For an arbitrary event $A$, denote by $(A)_{*}$ and $(A)^{*}$ the events of the finite Boolean algebra $\mathcal{A}$ with set of atoms $\mathcal{C}_{\mathcal{A}}$, defined as

$$
\begin{aligned}
(A)_{*} & =\bigvee\left\{C_{r} \in \mathcal{C}_{\mathcal{A}}: C_{r} \subseteq A\right\}, \\
(A)^{*} & =\bigvee\left\{C_{r} \in \mathcal{C}_{\mathcal{A}}: C_{r} \wedge A \neq \emptyset\right\}
\end{aligned}
$$

Obviously, if $A \in \mathcal{A}$ then $(A)_{*}=(A)^{*}=A$.

Given a full conditional probability $P(\cdot \mid \cdot)$ on a finite Boolean algebra $\mathcal{A}$, the bounds of its possible extensions to a new $E \mid H$ are such that $\underline{P}(E \mid H)=1$ if $E \wedge H=H$, otherwise it holds

$$
\underline{P}(E \mid H)=P\left((E \wedge H)_{*} \mid(E \wedge H)_{*} \vee\left(E^{c} \wedge H\right)^{*}\right),
$$

while $\bar{P}(E \mid H)=0$ if $E \wedge H=\emptyset$, otherwise it holds

$$
\bar{P}(E \mid H)=P\left((E \wedge H)^{*} \mid(E \wedge H)^{*} \vee\left(E^{c} \wedge H\right)_{*}\right) \text {. }
$$

In particular, the bounds for the extensions of an unconditional probability $P(\cdot)$ on $\mathcal{A}$ to a new $E$ reduce to $\underline{P}(E)=P\left((E)_{*}\right)$ and $\bar{P}(E)=P\left((E)^{*}\right)$.

Note that equations (3) and (4) are based on the "maximal" and the "minimal" conditional events in $\mathcal{A} \times \mathcal{A}^{0}$ implying $E \mid H$ and implied by $E \mid H$, respectively, with respect to the implication relation among conditional events $\subseteq_{G N}$ introduced by Goodman and Nguyen in [18]:

$$
E|H \subseteq G N F| K \Longleftrightarrow\left\{\begin{array}{l}
E \wedge H \subseteq F \wedge K, \\
E^{c} \wedge H \supseteq F^{c} \wedge K .
\end{array}\right.
$$

The set $\mathcal{P}=\{\tilde{P}(\cdot \mid \cdot)\}$ of all the conditional probabilities extending $P$ on $\left\langle\left\langle\mathcal{G}^{\prime}\right\rangle\right\rangle$ is a compact subset of the space $[0,1]^{\left\langle\left\langle\mathcal{G}^{\prime}\right\rangle\right\rangle}$ endowed with the product topology of pointwise convergence. Thus, the lower and upper envelopes of the extensions are defined as

$$
\underline{P}=\min \mathcal{P} \quad \text { and } \quad \bar{P}=\max \mathcal{P},
$$

where the minimum and the maximum are intended pointwise on the elements of $\left\langle\left\langle\mathcal{G}^{\prime}\right\rangle\right\rangle$. The functions $\underline{P}$ and $\bar{P}$ on $\left\langle\left\langle\mathcal{G}^{\prime}\right\rangle\right\rangle$ are known as lower and upper conditional probabilities, respectively [5]. Since every conditional probability is monotone with respect to $\subseteq_{G N}$ relation, it easily follows that

$$
E\left|H \subseteq_{G N} F\right| K \Longrightarrow\left\{\begin{array}{l}
P(E \mid H) \leq \underline{P}(F \mid K), \\
\bar{P}(E \mid H) \leq \bar{P}(F \mid K) .
\end{array}\right.
$$

\section{Conditioning for possibility measures}

In this section we assume $\mathcal{A}^{\prime}$ is a finite Boolean algebra with set of atoms $\mathcal{C}_{\mathcal{A}^{\prime}}=\left\{D_{1}, \ldots, D_{m}\right\}$.

A possibility measure $\Pi$ on $\mathcal{A}^{\prime}$ is a normalized maxitive function [14], i.e., it satisfies $\Pi(\emptyset)=0$, $\Pi(\Omega)=1$ and $\Pi(A \vee B)=\max \{\Pi(A), \Pi(B)\}$, for every $A, B \in \mathcal{A}^{\prime}$.

Every possibility measure $\Pi$ has an associated dual necessity measure $N$, defined for every $E \in \mathcal{A}^{\prime}$ as $N(E)=1-\Pi\left(E^{c}\right)$, which is minitive, i.e., for every $A, B \in \mathcal{A}^{\prime}, N(A \wedge B)=\min \{N(A), N(B)\}$.

Possibility measures are particular plausibility functions [20] and so particular upper probabilities $[15,10]$. They are completely characterized by their Möbius inversion $m_{\Pi}: \mathcal{A}^{\prime} \rightarrow[0,1]$ computed for every $E \in \mathcal{A}^{\prime}$ as

$$
m_{\Pi}(E)=\sum_{B \subseteq E}(-1)^{\left|\mathcal{C}_{E \backslash B}\right|} N(B),
$$

where $\mathcal{C}_{E \backslash B}=\left\{D_{r} \in \mathcal{C}_{\mathcal{A}^{\prime}}: D_{r} \subseteq E \wedge B^{c}\right\}$, which singles out the corresponding set of focal elements

$$
\mathbf{F}_{\Pi}=\left\{E \in \mathcal{A}^{\prime}: m_{\Pi}(E)>0\right\} .
$$

A coherence concept, similar to that given in the probabilistic setting [11], has been introduced in [7] for possibility assessments on finites spaces. In particular, in [7] it has been proved that coherence of a possibility assessment ensures its extendibility to 
every finite superset $\mathcal{E}^{\prime}$ of events, and for any event $E \in \mathcal{E}^{\prime}$ the coherent possibility values belong to a closed interval $[\underline{\Pi}(E), \bar{\Pi}(E)]$.

Various definitions of conditional possibility have been introduced, mainly by analogy with the Kolmogorovian probabilistic framework or by using some suitable conditioning rule (see, e.g., [9, 14]). All these definitions have in common the fact that a conditional measure is obtained as a derived concept of an "unconditional" possibility $\Pi(\cdot)$ on $\mathcal{A}^{\prime}$. For example, in the Kolmogorov-like definition, for $E \mid H \in \mathcal{A}^{\prime} \times \mathcal{A}^{\prime 0}$, the conditional possibility $\Pi(E \mid H)$ is defined as a solution of the equation in $x$

$$
\Pi(E \wedge H)=T(x, \Pi(H)),
$$

where $T$ is a t-norm. Notice that continuity of $T$ assures only the solvability of (10), while to get a unique solution a further constraint must be imposed. In this line, the Dubois and Prade's minimum specificity principle [14] always chooses the greatest solution by setting $\Pi_{D P}(E \mid H)=\Pi(H) \rightarrow_{T}$ $\Pi(E \wedge H)$, where $\rightarrow_{T}$ is the residuum operator associated to $T$.

In $[1,7]$ a general notion of $T$-conditional possibility (with $T$ any continuous t-norm) has been introduced as a primitive concept: the $T$-conditional possibility is directly defined as a function $\Pi(\cdot \mid \cdot)$ on a structured set of conditional events which satisfies a suitable set of axioms. The conditional possibilities obtained through the minimum specificity principle turn out to be particular $T$-conditional possibilities [2], while, in general $T$-conditional possibilities are not upper conditional probabilities.

On the other hand, a distinguished example of conditioning rule is the following one (see, e.g., [12, $17,16])$ defining, for $E \mid H \in \mathcal{A}^{\prime} \times \mathcal{A}^{\prime 0}$ with $N(H)>$ 0 ,

$$
\Pi_{B}(E \mid H)=\frac{\Pi(E \wedge H)}{\Pi(E \wedge H)+N\left(E^{c} \wedge H\right)} .
$$

Notice that the ratio in (11) could be properly defined also in case $N(H)=0$ : indeed, it is sufficient to have $\Pi(E \wedge H)+N\left(E^{c} \wedge H\right)>0$.

Previous rule has a strong connection with upper conditional probability and goes under the name of Bayesian conditioning rule [16]. Indeed, every possibility $\Pi$ induces a closed convex set of probabilities on $\mathcal{A}^{\prime}$ defined as $\mathcal{P}_{\Pi}=\left\{\tilde{P}: \mathcal{A}^{\prime} \rightarrow[0,1]\right.$ : $N \leq \tilde{P} \leq \Pi\}$ for which it holds $\Pi_{B}(E \mid H)=$ $\max \left\{\frac{\tilde{P}(E \wedge H)}{\tilde{P}(H)}: \tilde{P} \in \mathcal{P}_{\Pi}\right\}$ when $N(H)>0$. In [16] it has been shown that for every $H \in \mathcal{A}^{\prime 0}$ with $N(H)>0, \Pi_{B}(\cdot \mid H)$ turns out to be a possibility measure on $\mathcal{A}^{\prime}$.

In [6] it is proved that every possibility measure $\Pi$ on a finite Boolean algebra $\mathcal{A}^{\prime}$ can be obtained through the extension on $\left\langle\mathcal{A} \cup \mathcal{A}^{\prime}\right\rangle$ of a probability measure $P_{0}$ defined on another finite Boolean algebra $\mathcal{A}$. Actually, the class $\mathcal{P}_{\Pi}$ is the set of extensions of $P_{0}$ on $\left\langle\mathcal{A} \cup \mathcal{A}^{\prime}\right\rangle$ restricted to $\mathcal{A}^{\prime}$, thus the
Bayesian conditioning rule is intimately related to the extension of a probability.

Nevertheless, the Bayesian conditioning rule fails on all the conditioning events $H$ for which $N(H)=$ 0 . Thus, the idea is to provide a generalized Bayesian conditioning rule relying on a linearly ordered class $\left\{\Pi_{0}, \ldots, \Pi_{k}\right\}$ of possibilities on $\mathcal{A}^{\prime}$ such that the resulting conditional possibility $\Pi_{B}(\cdot \mid \cdot)$ can be obtained through the extension of a full conditional probability $P(\cdot \mid \cdot)$ defined on a different finite Boolean algebra $\mathcal{A}$.

Definition 1. A linearly ordered class of possibility measures $\left\{\Pi_{0}, \ldots, \Pi_{k}\right\}$ on $\mathcal{A}^{\prime}$ with sets of focal elements $\left\{\mathbf{F}_{\Pi_{0}}, \ldots, \mathbf{F}_{\Pi_{k}}\right\}$, is a covering class (or C-class for short) if $\bigvee_{E \in \bigcup_{\alpha=0}^{k}} \mathbf{F}_{\Pi_{\alpha}} E=\Omega$.

A linearly ordered class of necessity measures $\left\{N_{0}, \ldots, N_{k}\right\}$ is a C-class if the dual possibility measures $\left\{\Pi_{0}, \ldots, \Pi_{k}\right\}$ are a C-class.

Definition 2. A function $\Pi_{B}$ on $\mathcal{A}^{\prime} \times \mathcal{A}^{\prime 0}$ is a full B-conditional possibility measure on $\mathcal{A}^{\prime}$ if there exists a $C$-class $\left\{\Pi_{0}, \ldots, \Pi_{k}\right\}$ of possibilities on $\mathcal{A}^{\prime}$ such that, for every $E \mid H \in \mathcal{A}^{\prime} \times \mathcal{A}^{\prime 0}$,

$\Pi_{B}(E \mid H)= \begin{cases}0 & \text { if } E \wedge H=\emptyset, \\ \frac{\Pi_{\alpha}(E \wedge H)}{\Pi_{\alpha}(E \wedge H)+N_{\alpha}\left(E^{c} \wedge H\right)} & \text { otherwise, }\end{cases}$

where $N_{\alpha}$ is the dual necessity of $\Pi_{\alpha}$ and $\alpha \in$ $\{0, \ldots, k\}$ is the minimum index such that $\Pi_{\alpha}(E \wedge$ $H)+N_{\alpha}\left(E^{c} \wedge H\right)>0$.

The dual conditional measure defined for every $E \mid H \in \mathcal{A}^{\prime} \times \mathcal{A}^{\prime 0}$ as $N_{B}(E \mid H)=1-\Pi_{B}\left(E^{c} \mid H\right)$ will be called a full $B$-conditional necessity measure.

By results proved in [3], any C-class of possibility measures $\left\{\Pi_{0}, \ldots, \Pi_{k}\right\}$ on $\mathcal{A}^{\prime}$ can be generated by a full conditional probability $P(\cdot \mid \cdot)$ defined on a different finite Boolean algebra $\mathcal{A}$. Indeed, $P(\cdot \mid \cdot)$ is uniquely represented by the agreeing class $\left\{P_{0}, \ldots, P_{k}\right\}$ and, for $\alpha=0, \ldots, k, \Pi_{\alpha}$ is the upper envelope of the extensions of $P_{\alpha}$ on $\left\langle\mathcal{A} \cup \mathcal{A}^{\prime}\right\rangle$ restricted to $\mathcal{A}^{\prime}$. Moreover, the upper envelope of the extensions of $P(\cdot \mid \cdot)$ on $\left\langle\left\langle\left(\mathcal{A} \times \mathcal{A}^{0}\right) \cup\left(\mathcal{A}^{\prime} \times \mathcal{A}^{\prime 0}\right)\right\rangle\right\rangle$ coincides with $\Pi_{B}$ on $\mathcal{A}^{\prime} \times \mathcal{A}^{\prime 0}$.

The problem is then to find conditions assuring that extending a full conditional probability $P(\cdot \mid \cdot)$ on $\mathcal{A}$ one gets a C-class of possibility measures $\left\{\Pi_{0}, \ldots, \Pi_{k}\right\}$ on $\mathcal{A}^{\prime}$.

\section{Weakly logically independent Boolean algebras}

In this section we recall the condition of weak logical independence introduced in [6]. Such condition assures that given two finite algebras $\mathcal{A}$ and $\mathcal{A}^{\prime}$, and a probability measure $P(\cdot)$ on $\mathcal{A}$, the upper envelope $\bar{P}(\cdot)$ of the extensions of $P(\cdot)$ on $\left\langle\mathcal{A} \cup \mathcal{A}^{\prime}\right\rangle$ is a possibility measure on $\mathcal{A}^{\prime}$. Then we can show that under this (logical) condition the upper envelope of the 
extensions of $P(\cdot \mid \cdot)$ on $\left\langle\left\langle\left(\mathcal{A} \times \mathcal{A}^{0}\right) \cup\left(\mathcal{A}^{\prime} \times \mathcal{A}^{\prime 0}\right)\right\rangle\right\rangle$ is a full B-conditional possibility on $\mathcal{A}^{\prime} \times \mathcal{A}^{\prime 0}$ according to Definition 2.

Let $\mathcal{L}=\left\{C_{1}, \ldots, C_{n}\right\}, \mathcal{L}^{\prime}=\left\{D_{1}, \ldots, D_{m}\right\}$ be two partitions of $\Omega$. For any $D_{j} \in \mathcal{L}^{\prime}$, let us consider the event $\left(D_{j}\right)^{*}$ of the algebra $\mathcal{A}=\langle\mathcal{L}\rangle$ defined as in (2).

Given two partitions $\mathcal{L}, \mathcal{L}^{\prime}$ of $\Omega$, for any $D_{j} \in \mathcal{L}^{\prime}$, we consider the corresponding $\left(D_{j}\right)^{*} \in \mathcal{A}$. When two partitions are logically independent (i.e., $C_{i} \wedge$ $D_{j} \neq \emptyset$ for all $\left.i, j\right)$ one has $\left(D_{j}\right)^{*}=\Omega$, for any $D_{j} \in \mathcal{L}^{\prime}$.

Definition 3. A finite partition $\mathcal{L}^{\prime}$ is weakly logically independent of another finite partition $\mathcal{L}$ (in symbol $\mathcal{L}^{\prime} \perp_{w} \mathcal{L}$ ) if, for any given $D_{j} \in \mathcal{L}^{\prime}$, every $D_{k} \in \mathcal{L}^{\prime}(k \neq j)$ satisfies at least one of the following conditions:

(i) $D_{k} \subseteq\left(D_{j}\right)^{*}$

(ii) $D_{k} \wedge C_{i} \neq \emptyset$ for any $C_{i} \subseteq\left(D_{j}\right)^{*}$.

The following characterization of weak logical independence has been proven in [6].

Theorem 1. Let $\mathcal{L}=\left\{C_{1}, \ldots, C_{n}\right\}$ and $\mathcal{L}^{\prime}=$ $\left\{D_{1}, \ldots, D_{m}\right\}$ be two partitions of $\Omega$. The following two statements are equivalent:

(i) $\mathcal{L}^{\prime} \perp_{w} \mathcal{L}$;

(ii) there exists a permutation $\sigma$ of $\{1, \ldots, m\}$ such that $\left(D_{\sigma(1)}\right)^{*} \subseteq \ldots \subseteq\left(D_{\sigma(m)}\right)^{*}=\Omega$.

The notion of weak logical independence has been extended to finite Boolean algebras in [6].

Definition 4. A finite Boolean algebra $\mathcal{A}^{\prime}$ is weakly logically independent of another finite Boolean algebra $\mathcal{A}$ (in symbol $\mathcal{A}^{\prime} \perp_{w} \mathcal{A}$ ) if and only if $\mathcal{C}_{\mathcal{A}^{\prime}} \perp_{w} \mathcal{C}_{\mathcal{A}}$, where $\mathcal{C}_{\mathcal{A}}^{\prime}$ and $\mathcal{C}_{\mathcal{A}}$ are the sets of atoms of $\mathcal{A}^{\prime}$ and $\mathcal{A}$, respectively.

Note that $\mathcal{A}^{\prime} \perp_{w} \mathcal{A}$ if and only if $\mathcal{A} \perp_{w} \mathcal{A}^{\prime}$, where in the two statements the operator $(\cdot)^{*}$ is intended to be computed with respect to $\mathcal{C}_{\mathcal{A}}$ and $\mathcal{C}_{\mathcal{A}^{\prime}}$, respectively.

Proposition 1. Let $\mathcal{A}$ and $\mathcal{A}^{\prime}$ be two weakly logically independent finite Boolean algebras, whose sets of atoms are $\mathcal{C}_{\mathcal{A}}=\left\{C_{1}, \ldots, C_{n}\right\}$ and $\mathcal{C}_{\mathcal{A}^{\prime}}=$ $\left\{D_{1}, \ldots, D_{m}\right\}$, respectively, and let $\sigma$ be the permutation of $\{1, \ldots, m\}$ in condition (ii) of Theorem 1. Then the following statements hold:

(a) for every $E \in \mathcal{A}^{\prime}$ it holds $(E)^{*}=\left(D_{\sigma(h)}\right)^{*}$, where $h=\max \left\{j: D_{\sigma(j)} \subseteq E\right\}$;

(b) for every $E, F \in \mathcal{A}^{\prime}$ either $(E \vee F)^{*}=(E)^{*} \supseteq$ $(F)^{*}$ or $(E \vee F)^{*}=(F)^{*} \supseteq(E)^{*}$;

(c) for every $E \in \mathcal{A}^{\prime}$ it holds $(E)_{*}=\left(\left(D_{\sigma(k)}\right)^{*}\right)^{c}$, where $k=\max \left\{j: D_{\sigma(j)} \subseteq E^{c}\right\}$.

Proof. Statement (a) is a direct consequence of Theorem 1. Statement (b) immediately follows from (a). To prove statement (c) it is sufficient to take into account the definition of $(E)_{*}$ and statement (a).

\section{Conditioning in possibility theory: a generalized Bayesian rule}

Starting from a full conditional probability $P(\cdot \mid \cdot)$ on a finite $\mathcal{A}$, consider a finite algebra $\mathcal{A}^{\prime}$ weakly logically independent of $\mathcal{A}$. The aim is to characterize the properties of the restriction on $\mathcal{A}^{\prime} \times \mathcal{A}^{\prime 0}$ of the lower and upper envelopes of the extensions of $P(\cdot \mid \cdot)$ on $\left\langle\left\langle\left(\mathcal{A} \times \mathcal{A}^{0}\right) \cup\left(\mathcal{A}^{\prime} \times \mathcal{A}^{\prime 0}\right)\right\rangle\right\rangle$.

For $E \mid H \in \mathcal{A}^{\prime} \times \mathcal{A}^{\prime 0}$, if $E \wedge H=H$, since the operators $(\cdot)_{*}$ and $(\cdot)^{*}$ are computed with respect to $\mathcal{C}_{\mathcal{A}}$, the event $(E \wedge H)_{*} \vee\left(E^{c} \wedge H\right)^{*}$ could reduce to the impossible event, while it must hold $\underline{P}(E \mid H)=$ $P(\Omega \mid \Omega)=1$.

Hence, in order to simplify notation, for every $E \mid H \in \mathcal{A}^{\prime} \times \mathcal{A}^{\prime 0}$ we can define the operator $(\cdot \cdot \cdot)$. setting $(E \mid H)_{\bullet}=\Omega \mid \Omega$ if $E \wedge H=H$, otherwise

$$
(E \mid H)_{\bullet}=(E \wedge H)_{*} \mid(E \wedge H)_{*} \vee\left(E^{c} \wedge H\right)^{*},
$$

which associates to each conditional event $E \mid H \in$ $\mathcal{A}^{\prime} \times \mathcal{A}^{\prime 0}$ a conditional event in $\mathcal{A} \times \mathcal{A}^{0}$ having probability equal to the lower probability of $E \mid H$.

An analogous operation $(\cdot \cdot \cdot)^{\bullet}$ can be defined by focusing on the upper conditional probability. In fact, it holds $\bar{P}(E \mid H)=P(\emptyset \mid \Omega)=0$ when $E \wedge H=$ $\emptyset$, while, in this case, the event $(E \wedge H)^{*} \vee\left(E^{c} \wedge\right.$ $H)_{*}$ could reduce to the impossible event, so we set $(E \mid H)^{\bullet}=\emptyset \mid \Omega$ if $E \wedge H=\emptyset$ and otherwise we set

$$
(E \mid H)^{\bullet}=(E \wedge H)^{*} \mid(E \wedge H)^{*} \vee\left(E^{c} \wedge H\right)_{*} .
$$

Let us stress that for every conditional event $E \mid H$ it holds $\emptyset\left|\Omega \subseteq_{G N} E\right| H \subseteq_{G N} \Omega \mid \Omega$.

Notice that the operators $(\cdot \mid \cdot) \bullet$ and $(\cdot \mid \cdot) \bullet$ are consistent with the lower and upper conditional probabilities on $\mathcal{A}^{\prime} \times \mathcal{A}^{\prime 0}$, indeed, if $E \wedge H=\emptyset$, then $\underline{P}(E \mid H)=\bar{P}(E \mid H)=P((E \mid H) \bullet)=P\left((E \mid H)^{\bullet}\right)=$ 0 and if $E \wedge H=H$, then $\underline{P}(E \mid H)=\bar{P}(E \mid H)=$ $P\left((E \mid H)_{\bullet}\right)=P\left((E \mid H)^{\bullet}\right)=1$, thus we can simply set for every conditional event $E \mid H \in \mathcal{A}^{\prime} \times \mathcal{A}^{\prime 0}$

$$
\begin{aligned}
& \underline{P}(E \mid H)=P((E \mid H) \bullet, \\
& \bar{P}(E \mid H)=P\left((E \mid H)^{\bullet}\right) .
\end{aligned}
$$

Next result, which generalizes Theorem 3 in [6] to the conditional case, shows that, if $\mathcal{A}$ and $\mathcal{A}^{\prime}$ are weakly logical independent, then there exists a form of nesting involving the relation $\subseteq_{G N}$.

Proposition 2. Let $\mathcal{A}$ and $\mathcal{A}^{\prime}$ be two finite Boolean algebras with sets of atoms $\mathcal{C}_{\mathcal{A}}=\left\{C_{1}, \ldots, C_{n}\right\}$ and $\mathcal{C}_{\mathcal{A}^{\prime}}=\left\{D_{1}, \ldots, D_{m}\right\}$. If $\mathcal{A}^{\prime} \perp_{w} \mathcal{A}$ then for every $H \in \mathcal{A}^{\prime 0}$ there exists a permutation $\tau$ of the indices $\{1, \ldots, m\}$ such that

$$
\left(D_{\tau(1)} \mid H\right)^{\bullet} \subseteq_{G N} \ldots \subseteq_{G N}\left(D_{\tau(m)} \mid H\right)^{\bullet} .
$$

Proof. Let $\sigma$ be the permutation of $\{1, \ldots, m\}$ in condition (ii) of Theorem 1 and let $h$ be the index such that $\left(H^{c}\right)^{*}=\left(D_{\sigma(h)}\right)^{*}$ (see statement $(a)$ of Proposition 1). Partition $\{1, \ldots, m\}$ in two sets 
$I_{1}=\left\{i_{1}, \ldots, i_{t}\right\}$ and $I_{2}=\left\{i_{t+1}, \ldots, i_{m}\right\}$ such that $D_{i_{r}} \wedge H=\emptyset$ for $r=1, \ldots, t$ and $D_{i_{r}} \subseteq H$ for $r=t+1, \ldots, m$. Assume $i_{t+1} \leq \ldots \leq i_{m}$. For every $j \in I_{1}$ one has $\left(D_{\sigma(j)} \mid H\right)^{\bullet}=\emptyset \mid \Omega$, so set $\tau(r)=\sigma\left(i_{r}\right)$ for $r=1, \ldots, t$. For every $j \in I_{2}$ one has $\left(D_{\sigma(j)} \wedge H\right)^{*}=\left(D_{\sigma(j)}\right)^{*}$. Then, from statement (c) of Proposition 1, $\left(D_{\sigma(j)}^{c} \wedge H\right)_{*}=\left(\left(D_{\sigma(k)}\right)^{*}\right)^{c}$, where $k=\max \{j, h\}$. Therefore, for every $i, j \in I_{2}$ with $i \leq j$ we have $\left(\left(D_{\sigma(j)}^{c} \wedge H\right)^{*}\right)^{c} \subseteq\left(\left(D_{\sigma(i)}^{c} \wedge H\right)^{*}\right)^{c}$ which implies $\left(D_{\sigma(i)} \mid H\right)^{\bullet} \subseteq_{G N}\left(D_{\sigma(j)} \mid H\right)^{\bullet}$. Hence, set $\tau(r)=\sigma\left(i_{r}\right)$ for $r=t+1, \ldots, m$.

Let us point out that in previous proposition the symmetry of $\perp_{w}$ also implies that for every $K \in \mathcal{A}^{0}$ there exists a permutation $\kappa$ of the indices $\{1, \ldots, n\}$ such that

$$
\left(C_{\kappa(1)} \mid K\right)^{\bullet} \subseteq_{G N} \ldots \subseteq_{G N}\left(C_{\kappa(n)} \mid K\right)^{\bullet}
$$

where, in this case, the operator $(\cdot \mid \cdot)^{\bullet}$ is computed with respect to the set of atoms $\mathcal{C}_{\mathcal{A}^{\prime}}$.

Next proposition investigates the relation among conditional events $(E \mid H)^{\bullet},(F \mid H)^{\bullet}$ and $(E \vee F \mid H)^{\bullet}$ where $E|H, F| H, E \vee F \mid H \in \mathcal{A}^{\prime} \times \mathcal{A}^{\prime 0}$.

Proposition 3. Let $\mathcal{A}$ and $\mathcal{A}^{\prime}$ be two finite Boolean algebras with sets of atoms $\mathcal{C}_{\mathcal{A}}=\left\{C_{1}, \ldots, C_{n}\right\}$ and $\mathcal{C}_{\mathcal{A}^{\prime}}=\left\{D_{1}, \ldots, D_{m}\right\}$. If $\mathcal{A}^{\prime} \perp_{w} \mathcal{A}$ then for every $H \in \mathcal{A}^{\prime 0}$ and $E, F \in \mathcal{A}^{\prime}$ one of the following conditions holds:

(i) $(F \mid H)^{\bullet} \subseteq_{G N}(E \mid H)^{\bullet}=(E \vee F \mid H)^{\bullet}$;

(ii) $(E \mid H)^{\bullet} \subseteq_{G N}(F \mid H)^{\bullet}=(E \vee F \mid H)^{\bullet}$.

Proof. If $E \wedge H=\emptyset$ or $F \wedge H=\emptyset$ the conclusion is trivial and the same holds if $E \wedge H=H$ or $F \wedge$ $H=H$. Thus assume $E \wedge H \neq \emptyset \neq F \wedge H$ and $E \wedge H \neq H \neq F \wedge H$ and consider the permutation $\sigma$ of $\{1, \ldots, m\}$ in condition (ii) of Theorem 1. From statement (b) of Proposition 1 we have that either $((E \vee F) \wedge H)^{*}=(E \wedge H)^{*} \supseteq(F \wedge H)^{*}$ or $((E \vee$ $F) \wedge H)^{*}=(F \wedge H)^{*} \supseteq(E \wedge H)^{*}$. Suppose the first relation holds and let $i \geq j$ be the indices such that $\left(D_{\sigma(i)}\right)^{*}=(E \wedge H)^{*}$ and $\left(D_{\sigma(j)}\right)^{*}=(F \wedge H)^{*}$. Let $h$ and $k$ be the indices such that $\left(D_{\sigma(h)}\right)^{*}=\left(E \vee H^{c}\right)^{*}$ and $\left(D_{\sigma(k)}\right)^{*}=\left(F \vee H^{c}\right)^{*}$.

It holds $\left(E \vee H^{c}\right)^{*}=(E \wedge H)^{*} \vee\left(H^{c}\right)^{*} \supseteq(F \wedge$ $H)^{*} \vee\left(H^{c}\right)^{*}=\left(F \vee H^{c}\right)^{*}$, thus it must be $h \geq k$ and $\left(D_{\sigma(h)}\right)^{*}=\left((E \vee F) \vee H^{c}\right)^{*}$. Then, from statement (c) of Proposition 1, it immediately follows $\left(F^{c} \wedge\right.$ $H)_{*} \supseteq\left((E \vee F)^{c} \wedge H\right)_{*}=\left(E^{c} \wedge H\right)_{*}$ and so statement (i) is proved. When $((E \vee F) \wedge H)^{*}=(F \wedge H)^{*} \supseteq$ $(E \wedge H)^{*}$, with analogous considerations one can prove that statement (ii) holds

We consider now the lower and upper envelopes $\underline{P}(\cdot \mid \cdot)$ and $\bar{P}(\cdot \mid \cdot)$ of the set of extensions $\mathcal{P}$ on $\langle\langle(\mathcal{A} \times$ $\left.\left.\left.\mathcal{A}^{0}\right) \cup\left(\mathcal{A}^{\prime} \times \mathcal{A}^{\prime 0}\right)\right\rangle\right\rangle$, focusing on their restrictions on $\mathcal{A}^{\prime} \times \mathcal{A}^{\prime 0}$. In next theorem we prove that for every $H \in \mathcal{A}^{\prime 0}, \underline{P}(\cdot \mid H)$ and $\bar{P}(\cdot \mid H)$ are, respectively, a minitive and a maxitive measure on $\mathcal{A}^{\prime}$.
Theorem 2. Let $\mathcal{A}$ and $\mathcal{A}^{\prime}$ be two finite Boolean algebras, $P(\cdot \mid \cdot)$ a full conditional probability on $\mathcal{A}$ and $\underline{P}(\cdot \mid \cdot)$ and $\bar{P}(\cdot \mid \cdot)$ the lower and upper envelopes of the class $\mathcal{P}$ of extensions of $P(\cdot \mid \cdot)$ on $\left\langle\left\langle\left(\mathcal{A} \times \mathcal{A}^{0}\right) \cup\right.\right.$ $\left.\left.\left(\mathcal{A}^{\prime} \times \mathcal{A}^{\prime 0}\right)\right\rangle\right\rangle$. If $\mathcal{A}^{\prime} \perp_{w} \mathcal{A}$ then, for every $H \in \mathcal{A}^{\prime 0}$, the following statements hold:

(i) $\underline{P}(\cdot \mid H)$ is a necessity measure on $\mathcal{A}^{\prime}$;

(ii) $\bar{P}(\cdot \mid H)$ is a possibility measure on $\mathcal{A}^{\prime}$.

Proof. By the duality between $\underline{P}(\cdot \mid H)$ and $\bar{P}(\cdot \mid H)$ for every $H \in \mathcal{A}^{\prime 0}$, it is sufficient to prove (ii).

For $H \in \mathcal{A}^{\prime 0}$, we have to show that $\bar{P}(\cdot \mid H)$ is a possibility measure on $\mathcal{A}^{\prime}$. It trivially holds that $\bar{P}(\emptyset \mid H)=0$ and $\bar{P}(\Omega \mid H)=1$ thus we only need to prove that $\bar{P}(\cdot \mid H)$ is maxitive on $\mathcal{A}^{\prime}$. For every $E, F \in \mathcal{A}^{\prime}$ by Proposition 3 we have $(F \mid H)^{\bullet} \subseteq G N$ $(E \mid H)^{\bullet}=(E \vee F \mid H)^{\bullet}$ or $(E \mid H)^{\bullet} \subseteq_{G N}(F \mid H)^{\bullet}=$ $(E \vee F \mid H)^{\bullet}$. Suppose without loss of generality that $(F \mid H)^{\bullet} \subseteq_{G N}(E \mid H)^{\bullet}=(E \vee F \mid H)^{\bullet}$ from which it follows $P\left((F \mid H)^{\bullet}\right) \leq P\left((E \mid H)^{\bullet}\right)=P\left((E \vee F \mid H)^{\bullet}\right)$, that is $\bar{P}(F \mid H) \leq \bar{P}(E \mid H)=\bar{P}(E \vee F \mid H)$ which implies $\bar{P}(E \vee F \mid H)=\max \{\bar{P}(E \mid H), \bar{P}(F \mid H)\}$ and this concludes the proof.

From previous results and Theorem 3 in [3] the next result immediately follows.

Theorem 3. Let $\mathcal{A}$ and $\mathcal{A}^{\prime}$ be two finite Boolean algebras with $\mathcal{A}^{\prime} \perp_{w} \mathcal{A}, P(\cdot \mid \cdot)$ a full conditional probability on $\mathcal{A}$ represented by the agreeing class $\left\{P_{0}, \ldots, P_{k}\right\}$ of probability measures on $\mathcal{A}$, and $\underline{P}(\cdot \mid \cdot)$ and $\bar{P}(\cdot \mid \cdot)$ the lower and upper envelopes of the set $\mathcal{P}$ of extensions of $P(\cdot \mid \cdot)$ on $\left\langle\left\langle\left(\mathcal{A} \times \mathcal{A}^{0}\right) \cup\left(\mathcal{A}^{\prime} \times\right.\right.\right.$ $\left.\left.\mathcal{A}^{\prime 0}\right)\right\rangle$. Let $\left\{\underline{P}_{0}, \ldots, \underline{P}_{k}\right\}$ and $\left\{\bar{P}_{0}, \ldots, \bar{P}_{k}\right\}$ be the classes of restrictions on $\mathcal{A}^{\prime}$ of the lower and upper envelopes of the extensions of each $P_{\alpha}$ on $\left\langle\mathcal{A} \cup \mathcal{A}^{\prime}\right\rangle$. Then $\left\{\underline{P}_{0}, \ldots, \underline{P}_{k}\right\}$ and $\left\{\bar{P}_{0}, \ldots, \bar{P}_{k}\right\}$ are dual $C$ classes of necessity and possibility measures on $\mathcal{A}^{\prime}$, moreover, $\underline{P}(\cdot \mid \cdot)$ and $\bar{P}(\cdot \mid \cdot)$ on $\mathcal{A}^{\prime} \times \mathcal{A}^{\prime 0}$ coincide with the full $B$-conditional necessity and possibility measures on $\mathcal{A}^{\prime}$ generated by them.

Next example shows an application of previous theorem.

Example 1. Let $\mathcal{A}, \mathcal{A}^{\prime}$ be two finite Boolean algebras with sets of atoms $\mathcal{C}_{\mathcal{A}}=\left\{C_{1}, C_{2}, C_{3}, C_{4}\right\}$ and $\mathcal{C}_{\mathcal{A}^{\prime}}=\left\{D_{1}, D_{2}, D_{3}\right\}$, respectively, with $C_{1} \wedge\left(D_{2} \vee\right.$ $\left.D_{3}\right)=\emptyset, C_{2} \wedge\left(D_{2} \vee D_{3}\right)=\emptyset, C_{3} \wedge D_{3}=\emptyset$. Since $\left(D_{1}\right)^{*}=\Omega,\left(D_{2}\right)^{*}=C_{3} \vee C_{4}$ and $\left(D_{3}\right)^{*}=C_{4}$, it follows $\mathcal{A}^{\prime} \perp_{w} \mathcal{A}$.

Consider the full conditional probability $P(\cdot \mid \cdot)$ on $\mathcal{A}$ represented by the agreeing class $\left\{P_{0}, P_{1}\right\}$ whose distributions on $\mathcal{C}_{\mathcal{A}}$ are

\begin{tabular}{c|cccc}
$\mathcal{C}_{\mathcal{A}}$ & $C_{1}$ & $C_{2}$ & $C_{3}$ & $C_{4}$ \\
\hline$P_{0}$ & $\frac{1}{2}$ & 0 & $\frac{1}{2}$ & 0 \\
$P_{1}$ & 0 & $\frac{3}{4}$ & 0 & $\frac{1}{4}$
\end{tabular}

The agreeing class $\left\{P_{0}, P_{1}\right\}$ induces the following $C$-class of necessity measures $\left\{\underline{\underline{P}}_{0}, \underline{P}_{1}\right\}$ on $\mathcal{A}^{\prime}$ with dual possibility measures $\left\{\bar{P}_{0}, \bar{P}_{1}\right\}$, where $D_{i j}=$ $D_{i} \vee D_{j}$ : 


\begin{tabular}{c|cccccccc}
$\mathcal{A}^{\prime}$ & $\emptyset$ & $D_{1}$ & $D_{2}$ & $D_{3}$ & $D_{12}$ & $D_{13}$ & $D_{23}$ & $\Omega$ \\
\hline$\underline{P}_{0}$ & 0 & $\frac{1}{2}$ & 0 & 0 & 1 & $\frac{1}{2}$ & 0 & 1 \\
$\bar{P}_{0}$ & 0 & 1 & $\frac{1}{2}$ & 0 & 1 & 1 & $\frac{1}{2}$ & 1 \\
\hline $\bar{P}_{1}$ & 0 & $\frac{3}{4}$ & 0 & 0 & $\frac{3}{4}$ & $\frac{3}{4}$ & 0 & 1 \\
$\bar{P}_{1}$ & 0 & 1 & $\frac{1}{4}$ & $\frac{1}{4}$ & 1 & 1 & $\frac{1}{4}$ & 1
\end{tabular}

The full B-conditional possibility measure induced by $\left\{\bar{P}_{0}, \bar{P}_{1}\right\}$ on $\mathcal{A}^{\prime} \times \mathcal{A}^{\prime 0}$ is then

\begin{tabular}{c|cccccccc}
$\mathcal{A}^{\prime}$ & $\emptyset$ & $D_{1}$ & $D_{2}$ & $D_{3}$ & $D_{12}$ & $D_{13}$ & $D_{23}$ & $\Omega$ \\
\hline $\bar{P}\left(\cdot \mid D_{1}\right)$ & 0 & 1 & 0 & 0 & 1 & 1 & 0 & 1 \\
$\bar{P}\left(\cdot \mid D_{2}\right)$ & 0 & 0 & 1 & 0 & 1 & 0 & 1 & 1 \\
$\bar{P}\left(\cdot \mid D_{3}\right)$ & 0 & 0 & 0 & 1 & 0 & 1 & 1 & 1 \\
$\bar{P}\left(\cdot \mid D_{12}\right)$ & 0 & 1 & $\frac{1}{2}$ & 0 & 1 & 1 & $\frac{1}{2}$ & 1 \\
$\bar{P}\left(\cdot \mid D_{13}\right)$ & 0 & 1 & 0 & 0 & 1 & 1 & 0 & 1 \\
$\bar{P}\left(\cdot \mid D_{23}\right)$ & 0 & 0 & 1 & 1 & 1 & 1 & 1 & 1 \\
$\bar{P}(\cdot \mid \Omega)$ & 0 & 1 & $\frac{1}{2}$ & 0 & 1 & 1 & $\frac{1}{2}$ & 1
\end{tabular}

Let us stress that $\mathcal{A}^{\prime} \perp_{w} \mathcal{A}$ is only a sufficient condition in order to get a C-class $\left\{\Pi_{0}, \ldots, \Pi_{k}\right\}$ of possibility measures (and so a full B-conditional possibility measure) on $\mathcal{A}^{\prime}$ through the extension of a full conditional probability defined on $\mathcal{A}$.

Example 2. Let $\mathcal{A}^{\prime}$ be the finite Boolean algebra with set of atoms $\mathcal{C}_{\mathcal{A}^{\prime}}=\left\{D_{1}, D_{2}, D_{3}\right\}$ and $\left\{\Pi_{0}, \Pi_{1}\right\}$ the C-class of possibility measures on $\mathcal{A}^{\prime}$, where $D_{i j}=D_{i} \vee D_{j}$ :

$$
\begin{array}{c|cccccccc}
\mathcal{A}^{\prime} & \emptyset & D_{1} & D_{2} & D_{3} & D_{12} & D_{13} & D_{23} & \Omega \\
\hline \Pi_{0} & 0 & 1 & 1 & 0 & 1 & 1 & 1 & 1 \\
\hline \Pi_{1} & 0 & 0 & \frac{2}{3} & 1 & \frac{2}{3} & 1 & 1 & 1
\end{array}
$$

Consider the finite Boolean algebra $\mathcal{A}$ with set of atoms $\mathcal{C}_{\mathcal{A}}=\left\{C_{1}, C_{2}, C_{3}\right\}$ such that $C_{1} \wedge D_{3}=\emptyset$, $C_{2} \wedge\left(D_{1} \vee D_{2}\right)=\emptyset$ and $C_{3} \wedge D_{1}=\emptyset$. Let $P(\cdot \mid \cdot)$ be the full conditional probability on $\mathcal{A}$ represented by the agreeing class $\left\{P_{0}, P_{1}\right\}$ whose distributions on $\mathcal{C}_{\mathcal{A}}$ are

\begin{tabular}{c|ccc}
$\mathcal{C}_{\mathcal{A}}$ & $C_{1}$ & $C_{2}$ & $C_{3}$ \\
\hline$P_{0}$ & 1 & 0 & 0 \\
$P_{1}$ & 0 & $\frac{1}{3}$ & $\frac{2}{3}$
\end{tabular}

Simple computations show that $P_{\alpha}$ gives rise to $\Pi_{\alpha}$ as upper envelope on $\mathcal{A}^{\prime}$, for $\alpha=0,1$. Nevertheless, it holds $\left(D_{1}\right)^{*}=C_{1},\left(D_{2}\right)^{*}=C_{1} \vee C_{3}$ and $\left(D_{3}\right)^{*}=C_{2} \vee C_{3}$ thus $\neg\left(\mathcal{A}^{\prime} \perp_{w} \mathcal{A}\right)$.

\section{References}

[1] B. Bouchon-Meunier, G. Coletti, and C. Marsala. Independence and possibilistic conditioning. Ann. of Math. and Art. Int., 35(1-4):107-123, 2002.

[2] G. Coletti, D. Petturiti, and B. Vantaggi. Possibilistic and probabilistic likelihood functions and their extensions: Common features and specific characteristics. Fuzzy Sets and Sys., 250:25-51, 2014.

[3] G. Coletti, D. Petturiti, and B. Vantaggi. Conditional belief functions as lower envelopes of conditional probabilities in a finite setting. Inf. Sci., (Under Review).
[4] G. Coletti and R. Scozzafava. From Conditional Events to Conditional Measures: A New Axiomatic Approach. Ann. of Math. and Art. Int., 32(1-4):373-392, 2001.

[5] G. Coletti and R. Scozzafava. Probabilistic Logic in a Coherent Setting, volume 15 of Trends in Logic. Kluwer Ac. Pub., Dordrecht/Boston/London, 2002.

[6] G. Coletti, R. Scozzafava, and B. Vantaggi. Inferential processes leading to possibility and necessity. Inf. Sci., 245:132-145, 2013.

[7] G. Coletti and B. Vantaggi. T-conditional possibilities: Coherence and inference. Fuzzy Sets and Sys., 160(3):306-324, 2009.

[8] G. Coletti and B. Vantaggi. Probabilistic reasoning in a fuzzy context. In L.A. Zadeh et al., editor, Recent Developments and New Directions in Soft Computing, volume 317 of Studies in Fuzziness and Soft Computing, pages $97-$ 115. Springer Int. Pub., 2014.

[9] G. de Cooman. Possibility theory II: Conditional possibility. Int. J. of Gen. Sys., 25(4):325-351, 1997.

[10] G. de Cooman and D. Aeyels. Supremum preserving upper probabilities. Inf. Sci., 118(14):173-212, 1999.

[11] B. de Finetti. Theory of Probability, volume 1-2. John Wiley \& Sons, London, New York, Sydney, Toronto, 1975.

[12] A.P. Dempster. Upper and Lower Probabilities Induced by a Multivalued Mapping. Ann. of Math. Stat., 38(2):325-339, 1967.

[13] L.E. Dubins. Finitely Additive Conditional Probabilities, Conglomerability and Disintegrations. Ann. of Prob., 3(1):89-99, 1975.

[14] D. Dubois and H. Prade. Possibility Theory: An Approach to Computerized Processing of Uncertainty. Plenum Press, New York and London, 1988.

[15] D. Dubois and H. Prade. When upper probabilities are possibility measures. Fuzzy Sets and Sys., 49(1):65-74, 1992.

[16] D. Dubois and H. Prade. Bayesian conditioning in possibility theory. Fuzzy Sets and Sys., 92(2):223-240, 1997.

[17] R. Fagin and J.Y. Halpern. A new approach to updating beliefs. In P.P. Bonissone et al., editor, Uncertainty in Artificial Intelligence, pages 347-374. Elsevier Sci. Pub., 1991.

[18] I.R. Goodman and H.T. Nguyen. Conditional objects and the modeling of uncertainties, volume 21 of Fuzzy Computing, pages 119-138. North Holland, Amsterdam, 1988.

[19] A. Rényi. On Conditional Probability Spaces Generated by a Dimensionally Ordered Set of Measures. Th. of Prob. E Its App., 1(1):55-64, 1956.

[20] G. Shafer. A Mathematical Theory of Evidence. Princeton University Press, Princeton, NJ, 1976. 